\title{
GLOBAL ATTRACTIVITY OF THE PERIODIC KOLMOGOROV SYSTEM
}

\author{
XIANZHANG WEN ${ }^{12}$ and ZHICHENG WANG ${ }^{2}$
}

(Received 31 July, 2000; revised 28 December, 2000)

\begin{abstract}
We study a periodic Kolmogorov model with $m$ predators and $n$ prey. By means of the comparison theorem and a Liapunov function, a set of easily verifiable sufficient conditions that guarantee the existence, uniqueness and global attractivity of the positive periodic solution is obtained. Finally, some suitable applications are given to illustrate that the conditions of the main theorem are feasible.
\end{abstract}

\section{Introduction}

The Kolmogorov system is a rudimentary model in mathematical ecology and has been extensively investigated and developed (see $[8,9]$ and references therein). But most of the literature requires that the systems be representable as autonomous differential equations. If we consider the effects of environmental factors, the assumption of periodicity of the parameters is both realistic and important.

Our main purpose in this paper is to study the asymptotic behaviour of the general periodic Kolmogorov predator-prey system. Moreover, competition among predator species and among prey species is simultaneously considered, that is, we will investigate the following nonautonomous Kolmogorov predator-prey system of differential equations:

$$
\begin{cases}\dot{x}_{i}(t)=x_{i}(t) F_{i}\left(t, x_{1}(t), \ldots, x_{n}(t), y_{1}(t), \ldots, y_{m}(t)\right), & i=1, \ldots, n, \\ \dot{y}_{j}(t)=y_{i}(t) G_{j}\left(t, x_{1}(t), \ldots, x_{n}(t), y_{1}(t), \ldots, y_{m}(t)\right), & j=1, \ldots, m,\end{cases}
$$

where $x_{i}(t)$ denotes the density of species $X_{i}$ at time $t$ and $y_{j}(t)$ denotes the density of species $Y_{j}$ at time $t$. To describe system (1.1), we give the following assumptions.

\footnotetext{
'Department of Mathematics, Hunan Normal University, 410081 Changsha, Hunan, P. R. China

${ }^{2}$ Department of Applied Mathematics, Hunan University, 410082 Changsha, Hunan, P. R. China; e-mail: zcwang@hnu.net.cn.

(C) Australian Mathematical Society 2003, Serial-fee code 1446-1811/03
} 
(H1) $F_{i}: R \times R_{+}^{n+m} \rightarrow R$, is continuous and periodic with respect to $t$, that is, there exists a positive constant $T$, such that $F_{i}(t+T, \cdot, \ldots, \cdot)=F_{i}(t, \cdot, \ldots, \cdot)$; $G_{j}: R \times R_{+}^{n+m} \rightarrow R$, is continuous and periodic with respect to $t$, that is, for the above positive constant $T, G_{j}(t+T, \cdot \ldots, \cdot)=G_{j}(t, \cdot, \ldots, \cdot)$. Moreover,

$$
F_{i}(t, 0, \ldots, 0)>0, \quad G_{j}(t, 0, \ldots, 0)<0, \quad i=1, \ldots, n, j=1, \ldots, m .
$$

(H2) $F_{i}\left(t, x_{1}, \ldots, x_{n}, y_{1}, \ldots, y_{m}\right)$ and $G_{j}\left(t, x_{1}, \ldots, x_{n}, y_{1}, \ldots, y_{m}\right)$ are continuously differentiable with respect to $\left(x_{1}, \ldots, x_{n}, y_{1}, \ldots, y_{m}\right) \in R_{+}^{n+m}$. Moreover,

$$
\begin{gathered}
\frac{\partial F_{i}}{\partial x_{s}}<0, \quad \frac{\partial F_{i}}{\partial y_{l}}<0, \quad \frac{\partial G_{j}}{\partial x_{s}}>0, \quad \frac{\partial G_{j}}{\partial y_{l}}<0, \\
(i=1, \ldots, n, j=1, \ldots, m, s=1, \ldots, n, l=1, \ldots, m),
\end{gathered}
$$

for $\left(x_{1}, \ldots, x_{n}, y_{1}, \ldots, y_{m}\right) \in E$, where $E$ is an arbitrary bounded set of $R_{+}^{n+m}$.

(H3) There exist positive constants $B_{i}, i=1, \ldots, n$, such that

$$
F_{i}\left(t, 0, \ldots, 0, B_{i}, 0, \ldots, 0,0, \ldots, 0\right)<0, \quad i=1, \ldots, n .
$$

Moreover, for arbitrary bounded positive constants $K_{1}, \ldots, K_{n}$, there exist positive constants $D_{j}=D_{j}\left(K_{1}, \ldots, K_{n}\right)>0, j=1, \ldots, m$, such that

$$
G_{j}\left(t, K_{1}, \ldots, K_{n}, 0, \ldots, 0, D_{j}, 0, \ldots, 0\right)<0, \quad j=1, \ldots, m .
$$

Wu and Zhao obtained the globally attractive almost periodic solution in the almost competition Kolmogorov system in [12]. Yang and Xu obtained a globally attractive periodic solution in the periodic Volterra-Lotka predator-prey system in [13]. In this paper, we will study the periodic Kolmogorov predator-prey system. As an application, we will consider some ecological systems.

The structure of this paper is as follows: in Section 2, we investigate the persistence of system (1.1) and derive a persistence result. In Section 3 we obtain sufficient conditions for the uniqueness and global attractivity of the periodic solution of (1.1). Finally, in Section 4 we give some suitable applications to illustrate that the conditions of the main theorem are feasible.

\section{Persistence}

Throughout this paper,

$$
u\left(t, u_{0}\right)=\left(x_{1}\left(t, u_{0}\right), \ldots, x_{n}\left(t, u_{0}\right), y_{1}\left(t, u_{0}\right), \ldots, y_{m}\left(t, u_{0}\right)\right)^{T}
$$

denotes the solution of (1.1) with initial condition

$$
u_{0}=\left(x_{10}, \ldots, x_{n 0}, y_{10}, \ldots, y_{m 0}\right)^{T} \in \operatorname{int}\left(R_{+}^{n+m}\right) .
$$

The following lemmas are required for the derivation of a persistence result, which follows directly from [12, Theorem 4.2]. 
LEMMA 2.1. If for some $i \in\{1,2, \ldots, n\}$,

$$
\int_{0}^{T} F_{i}(t, 0, \ldots, 0) d t>0
$$

then the equation $d x_{i} / d t=x_{i} F_{i}\left(t, 0, \ldots, 0, x_{i}, 0, \ldots, 0\right)$ admits a globally asymptotically stable positive $T$-periodic solution $\bar{x}_{i}^{*}(t)$ in $\operatorname{int}\left(R_{+}\right)$.

LEMMA 2.2. If (P1) holds for all $i=1, \ldots, n$, and for some $j \in\{1,2, \ldots, m\}$,

$$
\int_{0}^{T} G_{j}\left(t, \bar{x}_{1}^{*}(t), \ldots, \bar{x}_{n}^{*}(t), 0, \ldots, 0\right) d t>0,
$$

then the equation $d y_{j} / d t=y_{j} G_{j}\left(t, \bar{x}_{1}^{*}, \ldots, \bar{x}_{n}^{*}, 0, \ldots, 0, y_{j}, 0, \ldots, 0\right)$ admits $a$ globally asymptotically stable positive $T$-periodic solution $\bar{y}_{j}^{*}(t)$ in $\operatorname{int}\left(R_{+}\right)$.

LEMMA 2.3. If (P1) holds for all $i=1, \ldots, n$, and (P2) holds for all $j=$ $1, \ldots, m$, and for some $i \in\{1, \ldots, n\}$,

$$
\int_{0}^{T} F_{i}\left(t, \bar{x}_{1}^{*}(t), \ldots, \bar{x}_{i-1}^{*}(t), 0, \bar{x}_{i+1}^{*}(t), \ldots, \bar{x}_{n}^{*}(t), \bar{y}_{1}^{*}(t), \ldots, \bar{y}_{m}^{*}(t)\right) d t>0,
$$

then the equation $d x_{i} / d t=x_{i} F_{i}\left(t, \bar{x}_{1}^{*}, \ldots, \bar{x}_{i-1}^{*}, x_{i}, \bar{x}_{i+1}^{*}, \ldots, \bar{x}_{n}^{*}, \bar{y}_{1}^{*}, \ldots, \bar{y}_{m}^{*}\right)$ admits a globally asymptotically stable positive $T$-periodic solution $\underline{x}_{i}^{*}(t)$ in $\operatorname{int}\left(R_{+}\right)$.

LEMMA 2.4. If (P1), (P3) hold for all $i=1, \ldots, n$, and (P2) holds for all $j=$ $1, \ldots, m$, and for some $j \in\{1, \ldots, m\}$,

$$
\int_{0}^{T} G_{j}\left(t, \underline{x}_{1}^{*}(t), \ldots, \underline{x}_{n}^{*}(t), \bar{y}_{1}^{*}(t), \ldots, \bar{y}_{j-1}^{*}(t), 0, \bar{y}_{j+1}^{*}(t), \cdots, \bar{y}_{m}^{*}(t)\right) d t>0,
$$

then the equation $d y_{j} / d t=y_{j} G_{j}\left(t, \underline{x}_{1}^{*}, \ldots, \underline{x}_{n}^{*}, \bar{y}_{1}^{*}, \ldots, \bar{y}_{j-1}^{*}, y_{j}, \bar{y}_{j+1}^{*}, \ldots, \bar{y}_{m}^{*}\right)$ admits a globally asymptotically stable positive $T$-periodic solution $\underline{y}_{j}^{*}(t)$ in $\operatorname{int}\left(R_{+}\right)$.

We can now obtain our main result of the section.

THEOREM 2.5. If (H1)-(H3) hold, (P1), (P3) hold for all $i=1, \ldots, n$, and (P2), (P4) hold for all $j=1, \ldots, m$, then system (1.1) is persistent, that is, there exist constants $M$ and $\eta, M>\eta>0$, such that for arbitrary

$$
u_{0}=\left(x_{10}, \ldots, x_{n 0}, y_{10}, \ldots, y_{m 0}\right)^{T} \in \operatorname{int}\left(R_{+}^{n+m}\right),
$$

there exists a $T\left(u_{0}\right)>0$, such that the solution $u\left(t, u_{0}\right)$ of $(1.1)$ satisfies

$$
\eta<x_{i}\left(t, u_{0}\right)<M, \quad \eta<y_{j}\left(t, u_{0}\right)<M,
$$

for all $t>T\left(u_{0}\right), i=1, \ldots, n, j=1, \ldots, m$. 
ProOF. We prove the theorem in two steps. Step (1). Solutions exist globally on $[0, \infty)$.

For any $u_{0}=\left(x_{10}, \ldots, x_{n 0}, y_{10}, \ldots, y_{m 0}\right) \in \operatorname{int}\left(R_{+}^{n+m}\right)$, let $I^{+}\left(u_{0}\right)=\left[0, \beta\left(u_{0}\right)\right)$ be the maximal interval of existence of $u\left(t, u_{0}\right)$. Then $x_{i}\left(t, u_{0}\right)>0, y_{j}\left(t, u_{0}\right)>0$ for $1 \leq i \leq n, 1 \leq j \leq m, t \in\left[0, \beta\left(u_{0}\right)\right)$. By the assumption $(\mathrm{H} 2), x_{i}\left(t, u_{0}\right)$ satisfies

$$
\frac{d x_{i}(t)}{d t} \leq x_{i}(t) F_{i}\left(t, 0, \ldots, 0, x_{i}(t), 0, \ldots, 0\right) .
$$

Therefore, by the comparison theorem, $0<x_{i}(t) \leq \bar{x}_{i}(t), t \in I^{+}\left(u_{0}\right)$, where $\bar{x}_{i}(t)$ is the unique solution of

$$
\frac{d x_{i}(t)}{d t}=x_{i}(t) F_{i}\left(t, 0, \ldots, 0, x_{i}(t), 0, \ldots, 0\right)
$$

with $\bar{x}_{i}(0)=x_{i 0}$. Since $\bar{x}_{i}(t)$ exists globally on $[0, \infty), x_{i}(t)$ exists globally on $[0, \infty)$. Moreover, for arbitrary $\delta>0$, there exists a $t_{0}>0$, such that $0<x_{i}(t)<\bar{x}_{i}^{*}(t)+\delta$, for all $t \geq t_{0}$.

By the assumption (H2), $y_{j}(t)$ satisfies

$$
\frac{d y_{j}(t)}{d t} \leq y_{j}(t) G_{j}\left(t, K_{1}, \ldots, K_{n}, 0, \ldots, 0, y_{j}(t), 0, \cdots, 0\right),
$$

where $K_{i}=\max \left\{\sup _{t \in[0, \infty)}\left[\bar{x}_{i}^{*}+\delta\right], \max _{t \in\left[0, t_{0}\right]} x_{i}(t)\right\}$. Therefore, by the comparison theorem, $0<y_{j}(t) \leq \bar{y}_{j}(t), t \in I^{+}\left(u_{0}\right)$, where $\bar{y}_{j}(t)$ is the unique solution of

$$
\frac{d y_{j}(t)}{d t}=y_{j}(t) G_{j}\left(t, K_{1}, \ldots, K_{n}, 0, \ldots, 0, y_{j}(t), 0, \ldots, 0\right),
$$

with $\bar{y}_{j}(0)=y_{j 0}$. Since $\bar{y}_{j}(t)$ exists globally on $[0, \infty), y_{j}(t)$ exists globally on $[0, \infty)$. Therefore $\beta\left(u_{0}\right)=\infty$ and $I^{+}\left(u_{0}\right)=[0, \infty)$.

Step (2). Uniform persistence.

By the first step, we know that for arbitrary $\delta>0$, there exists a $t_{0}>0$, such that $0<x_{i}(t)<\bar{x}_{i}^{*}+\delta, 1 \leq i \leq n$ for $t \geq t_{0}$. Now we prove that $y_{j}(t)$ is bounded for $1 \leq j \leq m$. Let $v_{i}(t)=x_{i}\left(t+t_{0}\right), v(t)=\left(v_{1}(t), \ldots, v_{n}(t)\right), v^{*}(t)=$ $\left(\bar{x}_{1}^{*}\left(t+t_{0}\right), \ldots, \bar{x}_{n}^{*}\left(t+t_{0}\right)\right)$ and $z_{j}(t)=y_{j}\left(t+t_{0}\right)$. Then

$$
\begin{aligned}
\frac{d z_{j}(t)}{d t} & =z_{j}(t) G_{j}\left(t+t_{0}, v_{1}(t), \ldots, v_{n}(t), z_{1}(t), \ldots, z_{m}(t)\right) \\
& \leq z_{j}(t) G_{j}\left(t+t_{0}, v_{1}(t), \ldots, v_{n}(t), 0, \ldots, 0, z_{j}(t), 0, \ldots, 0\right) \\
& \leq z_{j}(t) G_{j}\left(t+t_{0}, \bar{x}_{1}^{*}\left(t+t_{0}\right)+\delta, \ldots, \bar{x}_{n}^{*}\left(t+t_{0}\right)+\delta, 0, \ldots, 0, z_{j}(t), 0, \ldots, 0\right) .
\end{aligned}
$$

Therefore, by the comparison theorem, $0<z_{j}(t) \leq w_{j}(t)$, where $w_{j}(t)$ is the solution of

$$
\begin{gathered}
\frac{d w_{j}(t)}{d t}=w_{j}(t) G_{j}\left(t+t_{0}, \bar{x}_{1}^{*}\left(t+t_{0}\right)+\delta, \ldots, \bar{x}_{n}^{*}\left(t+t_{0}\right)+\delta\right. \\
\left.0, \ldots, 0, w_{j}(t), 0, \ldots, 0\right)
\end{gathered}
$$


with $w_{i}(0)=y_{j}\left(t_{0}\right)>0$. Choose $\delta_{0}>0$ small enough so that

$$
\int_{0}^{T} G_{j}\left(t, \bar{x}_{1}^{*}(t)+\delta, \ldots, \bar{x}_{n}^{*}(t)+\delta, 0, \ldots, 0\right) d t>0
$$

as $0<\delta<\delta_{0}$. Then (2.1) admits a unique globally asymptotically stable positive periodic solution $\bar{w}_{j}^{*}(t)$ in int $\left(R_{+}\right)$. For arbitrary $\varepsilon>0$, there exists a $t_{1}^{\prime}=t\left(y_{j}(0)\right)>$ 0 , such that $0<w_{j}(t)<\bar{w}_{j}^{*}(t)+\varepsilon / 2$ for $t \geq t_{1}^{\prime}$.

Considering the system

$$
\frac{d \bar{z}_{j}(t)}{d t}=\bar{z}_{j}(t) G_{j}\left(t+t_{0}, \bar{x}_{1}^{*}\left(t+t_{0}\right), \ldots, \bar{x}_{n}^{*}\left(t+t_{0}\right), 0, \ldots, 0, \bar{z}_{j}(t), 0, \ldots, 0\right),
$$

then $\lim _{t \rightarrow+\infty}\left(\bar{z}_{j}(t)-\bar{y}_{j}^{*}\left(t+t_{0}\right)\right)=0$, where $\bar{z}_{j}(t)$ is a solution of $(2.2)$ with $\bar{z}_{j}(0)=$ $y\left(t_{0}\right)>0$. Since the solution of $(2.1)$ is continuous with respect to parameter $\delta, \bar{w}_{j}^{*}(t)$ and $\bar{y}_{j}^{*}\left(t+t_{0}\right)$ are periodic solutions of (2.1) and (2.2) respectively. Hence, for the above $\varepsilon$, there exists a $\delta_{1}, 0<\delta_{1}<\delta_{0}$, such that $\left|\bar{y}_{j}^{*}\left(t+t_{0}\right)-\bar{w}_{j}^{*}(t)\right|<\varepsilon / 2$ for $0<\delta<\delta_{1}$. Therefore

$$
0<z_{j}(t) \leq w_{j}(t)<\bar{w}^{*}(t)+\varepsilon / 2<\bar{y}_{j}^{*}\left(t+t_{0}\right)+\varepsilon, \quad t \geq t_{1}^{\prime}, \delta<\delta_{1} .
$$

Accordingly, $0<y_{j}(t)<\bar{y}_{j}^{*}(t)+\varepsilon, t \geq t_{1}=t_{1}^{\prime}+t_{0}$.

Let $u_{i}(t)=x_{i}\left(t+t_{1}\right), i=1, \ldots, n$. Then $u_{i}(t)$ satisfies the inequality

$$
\begin{gathered}
\frac{d u_{i}(t)}{d t} \geq u_{i}(t) F_{i}\left(t+t_{1}, \bar{x}_{1}^{*}\left(t+t_{1}\right)+\delta, \ldots, \bar{x}_{i-1}^{*}\left(t+t_{1}\right)+\delta, u_{i}(t),\right. \\
\bar{x}_{i+1}^{*}\left(t+t_{1}\right)+\delta, \ldots, \bar{x}_{n}^{*}\left(t+t_{1}\right)+\delta, \\
\left.\bar{y}_{1}^{*}\left(t+t_{1}\right)+\varepsilon, \ldots, \bar{y}_{m}^{*}\left(t+t_{1}\right)+\varepsilon\right) .
\end{gathered}
$$

Therefore, by the comparison theorem, $u_{i}(t) \geq \underline{u}_{i}(t), t \geq 0,1 \leq i \leq n$, where $\underline{u}_{i}(t)$ is the unique solution of

$$
\begin{gathered}
\frac{d u_{i}(t)}{d t}=u_{i}(t) F_{i}\left(t+t_{1}, \bar{x}_{1}^{*}\left(t+t_{1}\right)+\delta, \ldots, \bar{x}_{i-1}^{*}\left(t+t_{1}\right)+\delta, u_{i}(t),\right. \\
\bar{x}_{i+1}^{*}\left(t+t_{1}\right)+\delta, \ldots, \bar{x}_{n}^{*}\left(t+t_{1}\right)+\delta, \\
\left.\bar{y}_{1}^{*}\left(t+t_{1}\right)+\varepsilon, \ldots, \bar{y}_{m}^{*}\left(t+t_{1}\right)+\varepsilon\right)
\end{gathered}
$$

with $\underline{u}_{i}(0)=u_{i}(0)=x_{i}\left(t_{1}\right)>0$. Define

$$
\begin{gathered}
\bar{F}_{i}\left(t+t_{1}, \delta, \varepsilon\right)=F_{i}\left(t+t_{1}, \bar{x}_{1}^{*}\left(t+t_{1}\right)+\delta, \ldots, \bar{x}_{i-1}^{*}\left(t+t_{1}\right)+\delta, 0,\right. \\
\bar{x}_{i+1}^{*}\left(t+t_{1}\right)+\delta, \ldots, \bar{x}_{n}^{*}\left(t+t_{1}\right)+\delta, \\
\left.\bar{y}_{1}^{*}\left(t+t_{1}\right)+\varepsilon, \ldots, \bar{y}_{m}^{*}\left(t+t_{1}\right)+\varepsilon\right) .
\end{gathered}
$$


There exist positive constants $\varepsilon_{1}$ and $\delta_{2}, \delta_{2}<\delta_{1}$, such that $\int_{0}^{T} \bar{F}_{i}\left(\xi+t_{1}, \delta, \varepsilon\right) d \xi>0$, when $0<\delta<\delta_{2}, 0<\varepsilon<\varepsilon_{1}, i=1, \ldots, n$. Therefore we have

$$
\lim _{i \rightarrow \infty}\left(\underline{u}_{i}(t)-u_{i}^{*}(t)\right)=0
$$

where $u_{i}^{*}(t)$ is the globally asymptotically stable positive periodic solution of (2.3). By (2.4), we know that for arbitrary $\gamma<\inf _{t \in R_{+}} \underline{u}_{i}^{*}(t), 1 \leq i \leq n$, there exists $t_{2}^{\prime}=t\left(x_{i}(0)\right)>0$, such that $\underline{u}_{i}(t)>u_{i}^{*}(t)-\gamma / 2, t>t_{1}, 1 \leq i \leq n$. Considering the equation

$$
\begin{array}{r}
\frac{d w_{i}(t)}{d t}=w_{i}(t) F_{i}\left(t+t_{1}, \bar{x}_{1}^{*}\left(t+t_{1}\right), \ldots, \bar{x}_{i-1}^{*}\left(t+t_{1}\right), w_{i}(t),\right. \\
\left.\bar{x}_{i+1}^{*}\left(t+t_{1}\right), \ldots, \bar{x}_{n}^{*}\left(t+t_{1}\right), \bar{y}_{1}^{*}\left(t+t_{1}\right), \ldots, \bar{y}_{m}^{*}\left(t+t_{1}\right)\right),
\end{array}
$$

we know that (2.5) admits a unique positive periodic solution $\underline{x}_{i}^{*}\left(t+t_{1}\right)$, which is globally asymptotically stable in $\operatorname{int}\left(R_{+}\right)$. This holds since the solution of (2.3) is continuous. with respect to parameters $\delta$ and $\varepsilon$, and $u_{i}^{*}(t)$ and $\underline{x}_{i}^{*}\left(t+t_{1}\right)$ are positive periodic solutions of (2.3) and (2.5), respectively. For the above $\gamma$, there exist constants $\varepsilon_{2}, \delta_{3}, 0<\delta_{3}<\delta_{2}, 0<\varepsilon_{2}<\varepsilon_{1}$, such that

$$
\left|\underline{x}_{i}^{*}\left(t+t_{1}\right)-u_{i}^{*}(t)\right|<\gamma / 2, \quad 0<\delta<\delta_{3}, 0<\varepsilon<\varepsilon_{2} .
$$

Hence, for $0<\delta<\delta_{3}, 0<\varepsilon<\varepsilon_{2}$ and $t \geq t_{2}^{\prime}$,

$$
u_{i}(t) \geq \underline{u}_{i}(t)>u_{i}^{*}(t)-\gamma / 2>\underline{x}_{i}^{*}\left(t+t_{1}\right)-\gamma
$$

Therefore, letting $t_{2}=t_{2}^{\prime}+t_{1}$, we obtain

$$
x_{i}(t) \geq \underline{x}_{i}^{*}(t)-\gamma, \quad t \geq t_{2}>0,1 \leq i \leq n .
$$

Similarly, for arbitrary $0<v<\inf _{t \in R} \underline{y}_{j}^{*}(t)$, there exists $t_{3}>t_{2}$, such that

$$
y_{j}(t) \geq \underline{y}_{j}^{*}(t)-v, \quad t \geq t_{3}>0,1 \leq j \leq m .
$$

By the above proof, we know that system (1.1) is persistent, which completes our proof.

\section{Global attractivity}

We first give an assumption and then introduce our main theorem. 
(H4) There exist continuously differentiable bounded functions $\beta_{i}:(0, \infty) \rightarrow(0, \infty)$, $\alpha_{j}:(0, \infty) \rightarrow(0, \infty), i=1, \ldots, n, j=1, \ldots, m$, and a positive $T$-periodic function $b(t): R_{+} \rightarrow R_{+}$, such that

$$
\begin{aligned}
& \frac{\partial \beta_{i}\left(x_{i}\right) F_{i}(t, x, y)}{\partial x_{i}}+\left[\sum_{l \neq i}^{n} \beta_{l}\left(x_{l}\right)\left|\frac{\partial F_{l}(t, x, y)}{\partial x_{i}}\right|+\sum_{s=1}^{m} \alpha_{s}\left(y_{s}\right)\left|\frac{\partial G_{s}(t, x, y)}{\partial x_{i}}\right|\right] \\
& \quad \leq-b(t), \\
& \frac{\partial \alpha_{j}\left(y_{j}\right) G_{j}(t, x, y)}{\partial y_{j}}+\left[\sum_{l=1}^{n} \beta_{l}\left(x_{l}\right)\left|\frac{\partial F_{l}(t, x, y)}{\partial y_{j}}\right|+\sum_{s \neq j}^{m} \alpha_{s}\left(y_{s}\right)\left|\frac{\partial G_{s}(t, x, y)}{\partial y_{j}}\right|\right] \\
& \leq-b(t)
\end{aligned}
$$

for all $t \in R_{+}$and $x=\left(x_{1}, \ldots, x_{n}\right), y=\left(y_{1}, \ldots, y_{m}\right), x_{i} \in[\eta, M], y_{j} \in[\eta, M]$, $i=1, \ldots, n, j=1, \ldots, m$.

THEOREM 3.1. If (H4) and the conditions of Theorem 2.5 hold, then (1.1) admits a globally attractive positive $T$-periodic solution $u^{*}(t) \in \operatorname{int}\left(R_{+}^{n+m}\right)$, that is, for any $u_{0} \in$ $\operatorname{int}\left(R_{+}^{n+m}\right)$, the solution $u(t)$ of $(1.1)$ with $u(0)=u_{0}$ satisfies $\lim _{t \rightarrow \infty}\left(u(t)-u^{*}(t)\right)=0$.

Proof. By Theorem 2.5, we know that system (1.1) is persistent, that is, there exist $M>\eta>0$, for any $u_{0}=\left(x_{10}, \ldots, x_{n 0}, y_{10}, \ldots, y_{m 0}\right)^{T} \in \operatorname{int}\left(R_{+}^{n+m}\right)$, there exists $T_{0}=T\left(u_{0}\right)>0$, such that the positive solution $u\left(t, u_{0}\right)$ of system (1.1) satisfies

$$
\eta<x_{i}\left(t, u_{0}\right)<M, \quad \eta<y_{j}\left(t, u_{0}\right)<M,
$$

for $t>T_{0}, i=1, \ldots, n, j=1, \ldots, m$. By [11], we know that system (1.1) admits a positive $T$-periodic solution $u^{*}(t)=\left(x_{1}^{*}(t), \ldots, x_{n}^{*}(t), y_{1}^{*}(t), \ldots, y_{m}^{*}(t)\right)^{T}$.

Define

$$
\begin{aligned}
V(t) & =V\left(u\left(t+T_{0}\right), u^{*}\left(t+T_{0}\right)\right) \\
& =\sum_{i=1}^{n}\left|\int_{x_{i}\left(t+T_{0}\right)}^{x_{i}^{*}\left(t+T_{0}\right)} \frac{\beta_{i}(w)}{w} d w\right|+\sum_{j=1}^{m}\left|\int_{y_{j}\left(t+T_{0}\right)}^{y_{j}^{*}\left(t+T_{0}\right)} \frac{\alpha_{j}(w)}{w} d w\right| .
\end{aligned}
$$

For any $w \in[\eta, M]$, we have $\beta_{i}(w) / w>0$ and $\alpha_{j}(w) / w>0$. Hence there exist positive constants $D_{1}$ and $D_{2}$, such that

$$
\begin{aligned}
D_{1} & {\left[\sum_{i=1}^{n}\left|x_{i}^{*}\left(t+T_{0}\right)-x_{i}\left(t+T_{0}\right)\right|+\sum_{j=1}^{m}\left|y_{j}^{*}\left(t+T_{0}\right)-y_{j}\left(t+T_{0}\right)\right|\right] } \\
& \leq V(t) \leq D_{2}\left[\sum_{i=1}^{n}\left|x_{i}^{*}\left(t+T_{0}\right)-x_{i}\left(t+T_{0}\right)\right|+\sum_{i=1}^{m}\left|y_{j}^{*}\left(t+T_{0}\right)-y_{j}\left(t+T_{0}\right)\right|\right] .
\end{aligned}
$$


Using the notation $t_{+}=t+T_{0}$ and letting $\eta\left(x_{i}^{*}, x_{i}\right)=s x_{i}^{*}\left(t_{+}\right)+(1-s) x_{i}\left(t_{+}\right)$, we have

$$
\begin{aligned}
\frac{d}{d t} & \int_{x_{i}\left(t_{+}\right)}^{x_{i}^{*}\left(t_{+}\right)} \frac{\beta_{i}(w)}{w} d w \\
= & \beta_{i}\left(x_{i}^{*}\left(t_{+}\right)\right) F_{i}\left(t_{+}, u^{*}\left(t_{+}\right)\right)-\beta_{i}\left(x_{i}\left(t_{+}\right)\right) F_{i}\left(t_{+}, u\left(t_{+}\right)\right) \\
= & \int_{0}^{1} \frac{d}{d s}\left[\beta_{i}\left(\eta\left(x_{i}^{*}, x_{i}\right)\right) F_{i}\left(t_{+}, \eta\left(u^{*}, u\right)\right)\right] d s \\
= & \int_{0}^{1}\left[\sum_{j=1}^{n} \frac{\partial\left(\beta_{i}\left(\eta\left(x_{i}^{*}, x_{i}\right)\right) F_{i}\left(t_{+}, \eta\left(u^{*}, u\right)\right)\right)}{\partial x_{j}}\left(x_{j}^{*}\left(t_{+}\right)-x_{j}\left(t_{+}\right)\right)\right. \\
& \left.+\sum_{j=1}^{m} \frac{\partial\left(\beta_{i}\left(\eta\left(x_{i}^{*}, x_{i}\right)\right) F_{i}\left(t_{+}, \eta\left(u^{*}, u\right)\right)\right)}{\partial y_{j}}\left(y_{j}^{*}\left(t_{+}\right)-y_{j}\left(t_{+}\right)\right)\right] d s \\
\frac{d}{d t} & \int_{y_{j}\left(t_{+}\right)}^{y_{j}^{*}\left(t_{+}\right)} \frac{\alpha_{j}(w)}{w} d w \\
= & \alpha_{j}\left(y_{j}^{*}\left(t_{+}\right)\right) G_{j}\left(t_{+}, u^{*}\left(t_{+}\right)\right)-\alpha_{j}\left(y_{j}\left(t_{+}\right)\right) G_{j}\left(t_{+}, u\left(t_{+}\right)\right) \\
= & \int_{0}^{1} \frac{d}{d s}\left[\alpha_{j}\left(\eta\left(y_{j}^{*}, y_{j}\right)\right) G_{j}\left(t_{+}, \eta\left(u^{*}, u\right)\right)\right] d s \\
= & \int_{0}^{1}\left[\sum_{r=1}^{n} \frac{\partial\left(\alpha_{j}\left(\eta\left(y_{j}^{*}, y_{j}\right)\right) G_{j}\left(t_{+}, \eta\left(u^{*}, u\right)\right)\right)}{\partial x_{r}}\left(x_{r}^{*}\left(t_{+}\right)-x_{r}\left(t_{+}\right)\right)\right. \\
& \left.\left.+\sum_{r=1}^{m} \frac{\partial\left(\alpha_{j}\left(\eta\left(y_{j}^{*}, y_{j}\right)\right) G_{j}\left(t_{+}, \eta\left(u^{*}, u\right)\right)\right)}{\partial y_{r}}\left(t_{+}\right)-y_{r}\left(t_{+}\right)\right)\right] d s .
\end{aligned}
$$

Therefore, calculating the upper right derivative $D^{+} V(t)$ of $V(t)$ along the solutions of (1.1), we get

$$
\begin{aligned}
D^{+} V(t) \leq & \int_{0}^{1}\left\{\sum _ { i = 1 } ^ { n } \left[\frac{\partial \beta_{i}\left(\eta\left(x_{i}^{*}, x_{i}\right)\right) F_{i}\left(t_{+}, \eta\left(u^{*}, u\right)\right)}{\partial x_{i}}\right.\right. \\
& +\left(\sum_{j \neq i}^{n} \beta_{j}\left(\eta\left(x_{j}^{*}, x_{j}\right)\right)\left|\frac{\partial F_{j}\left(t_{+}, \eta\left(u^{*}, u\right)\right)}{\partial x_{i}}\right|\right. \\
& \left.\left.\left.+\sum_{j=1}^{m} \alpha_{j}\left(\eta\left(y_{j}^{*}, y_{j}\right)\right)\left|\frac{\partial G_{j}\left(t_{+}, \eta\left(u^{*}, u\right)\right)}{\partial x_{i}}\right|\right)\right]\left|x_{i}^{*}\left(t_{+}\right)-x_{i}\left(t_{+}\right)\right|\right\} d s \\
& +\int_{0}^{1}\left\{\sum _ { j = 1 } ^ { m } \left[\frac{\partial \alpha_{j}\left(\eta\left(y_{j}^{*}, y_{j}\right)\right) G_{j}\left(t_{+}, \eta\left(u^{*}, u\right)\right)}{\partial y_{j}}\right.\right.
\end{aligned}
$$




$$
\begin{aligned}
& +\left(\sum_{r=1}^{n} \beta_{r}\left(\eta\left(x_{r}^{*}, x_{r}\right)\right)\left|\frac{\partial F_{r}\left(t_{+}, \eta\left(u^{*}, u\right)\right)}{\partial y_{j}}\right|\right. \\
& \left.\left.\left.+\sum_{r \neq j}^{m} \alpha_{r}\left(\eta\left(y_{r}^{*}, y_{r}\right)\right)\left|\frac{\partial G_{r}\left(t_{+}, \eta\left(u^{*}, u\right)\right)}{\partial y_{j}}\right|\right)\right]\left|y_{j}^{*}\left(t_{+}\right)-y_{j}\left(t_{+}\right)\right|\right\} d s \\
& \leq-b\left(t_{+}\right)\left(\sum_{i=1}^{n}\left|x_{i}^{*}\left(t_{+}\right)-x_{i}\left(t_{+}\right)\right|+\sum_{j=1}^{m}\left|y_{j}^{*}\left(t_{+}\right)-y_{j}\left(t_{+}\right)\right|\right) \leq-\frac{b\left(t_{+}\right)}{D_{2}} V(t) .
\end{aligned}
$$

Therefore, by the comparison theorem, we get

$$
V(t) \leq V\left(u^{*}\left(T_{0}\right), u\left(T_{0}\right)\right) e^{-\int_{0}^{\prime}\left(b\left(s+T_{0}\right) / D_{2}\right) d s},
$$

since $b(t)$ is a positive periodic function. This implies that $\lim _{t \rightarrow \infty} V(t)=0$. Accordingly, we have

$$
\lim _{t \rightarrow \infty}\left|x_{i}(t)-x_{i}^{*}(t)\right|=0, \quad i=1, \ldots, n,
$$

and

$$
\lim _{t \rightarrow \infty}\left|y_{j}(t)-y_{j}^{*}(t)\right|=0, \quad j=1, \ldots, m .
$$

This completes the proof of Theorem 3.1.

\section{Applications}

In this section, some suitable applications are given to illustrate that the conditions of Theorem 3.1 are feasible.

We first consider the system

$$
\left\{\begin{array}{l}
\dot{x}_{i}(t)=x_{i}(t)\left[b_{i}(t)-\sum_{s=1}^{n} a_{i s}(t) x_{s}(t)-\sum_{s=1}^{m} c_{i s}(t) y_{s}(t)\right], \\
\dot{y}_{j}(t)=y_{j}(t)\left[-r_{j}(t)+\sum_{s=1}^{n} d_{j s}(t) x_{s}(t)-\sum_{s=1}^{m} e_{j s}(t) y_{s}(t)\right],
\end{array}\right.
$$

where $b_{i}(t), r_{j}(t), a_{i j}(t), c_{i j}(t), d_{i j}(t), e_{i j}(t)$ are positive continuous $T$-periodic functions with respect to $t$. There exists a positive constant $\eta_{0}>0$, such that $\min _{t \in R^{+}}\left\{b_{i}(t), a_{i i}(t), e_{j j}(t)\right\}>\eta_{0}$.

Letting $\beta_{i}(\cdot)=1, \alpha_{j}(\cdot)=1, i=1, \ldots, n, j=1, \ldots, m$ in the proof of Theorem 3.1, we obtain the following theorem.

THEOREM 4.1. If system (4.1) is persistent, and there exists a continuous positive periodic function $b(t)$, with $\int_{0}^{T} b(s) d s>0$ such that

$$
\left\{\begin{array}{l}
a_{i i}(t)-\left[\sum_{s \neq i}^{n} a_{s i}(t)+\sum_{k=1}^{m} d_{s i}(t)\right]>b(t), \\
e_{j j}(t)-\left[\sum_{s=1}^{n} c_{s j}(t)+\sum_{s \neq j}^{m} e_{s j}(t)\right]>b(t)
\end{array}\right.
$$


for all $i=1, \ldots, n, j=1, \ldots, m$, then there exists a unique strictly positive periodic solution in the system (4.1) which is globally attracting.

In the following, we consider a simple system

$$
\left\{\begin{array}{l}
\dot{x}_{1}(t)=x_{1}(t)\left(r_{1}-a_{11} x_{1}(t)-a_{12} x_{2}(t)\right) \\
\dot{x}_{i}(t)=x_{i}(t)\left(-r_{i}+a_{i, i-1} x_{i-1}(t)-a_{i i} x_{i}(t)-a_{i, i+1} x_{i+1}(t)\right), 2 \leq i \leq n-1, \\
\dot{x}_{n}(t)=x_{n}(t)\left(-r_{n}+a_{n, n-1} x_{n-1}(t)-a_{n n} x_{n}(t)\right),
\end{array}\right.
$$

where $r_{i}, a_{i j}, 1 \leq i \leq n, 1 \leq j \leq n$, are all positive constants.

Define $D_{1}=r_{1}, \Delta_{i}=\prod_{j=1}^{j=i} \bar{a}_{j j}$,

$$
D_{i}=\left|\begin{array}{ccccc}
a_{11} & 0 & \ldots & 0 & r_{1} \\
-a_{21} & a_{22} & \cdots & 0 & -r_{2} \\
0 & -a_{32} & \ddots & 0 & -r_{3} \\
\vdots & \vdots & \ddots & \ddots & \vdots \\
0 & 0 & \ldots & -a_{i, i-1} & -r_{i}
\end{array}\right|, \quad i=2, \ldots, n,
$$

and also define $E_{1}=r_{1}-a_{12} D_{2} / \Delta_{2}, a_{n, n+1}=0$ and, for $i=2, \ldots, n$,

$$
E_{i}=\left|\begin{array}{ccccc}
a_{11} & 0 & \cdots & 0 & r_{1}-a_{12} D_{2} / \Delta_{2} \\
-a_{21} & a_{22} & \cdots & 0 & -r_{2}-a_{23} D_{3} / \Delta_{3} \\
0 & -a_{32} & \ddots & 0 & -r_{3}-a_{34} D_{4} / \Delta_{4} \\
\vdots & \vdots & \ddots & \ddots & \vdots \\
0 & 0 & \cdots & -a_{i, i-1} & -r_{i}-a_{i, i+1} D_{i+1} / \Delta_{i+1}
\end{array}\right| .
$$

We then have the following theorem

THEOREM 4.2. If $D_{i}>0$ and $E_{i}>0$, for $i=1, \ldots, n$, and

$$
\begin{aligned}
a_{11} & >a_{21}, \quad a_{n n}>a_{n-1, n}, \\
a_{i i} & >a_{i-1, i}+a_{i+1, i}, \quad \text { for } i=2, \ldots, n-1,
\end{aligned}
$$

then the positive steady state of system (4.2) is globally attracting in int $\left(R_{+}^{n}\right)$.

PROOF. In fact

$$
\begin{aligned}
& \bar{x}_{i}^{*}=\frac{D_{i}}{\Delta_{i}}>0, \quad \text { for } i=1, \ldots, n, \\
& \underline{x}_{i}^{*}=\frac{E_{i}}{\Delta_{i}}>0, \quad \text { for } i=1, \ldots, n-1 .
\end{aligned}
$$

By Theorem 2.5, we know that system (4.2) is persistent. Finally, by (4.5) and (4.6), we know that assumption (H4) of Theorem 3.1 holds, which completes the proof. 
In particular, we consider

$$
\left\{\begin{array}{l}
\dot{x}(t)=x(t)\left(r_{1}-a_{11} x(t)-a_{12} y(t)\right) \\
\dot{y}(t)=y(t)\left(-r_{2}+a_{21} x(t)-a_{22} y(t)\right),
\end{array}\right.
$$

where $r_{i}, a_{i j}, i, j=1,2$ are all positive constants.

COROLLARY 4.3 ([9]). If $a_{11} a_{22}-a_{12} a_{21}>0$, then the positive steady state of system (4.7) is globally attracting.

PROOF. In fact, $r_{1} a_{21}-r_{2} a_{11}>0$ is a necessary condition for system (4.7) if there exists a positive steady state in the system since

$$
\begin{array}{ll}
\bar{x}^{*}=\frac{r_{1}}{a_{11}}>0, & \underline{x}^{*}=\frac{\left(a_{11} a_{22}-a_{12} a_{21}\right) r_{1}+a_{11} a_{12} r_{2}}{a_{11}^{2} a_{22}}>0, \\
\bar{y}^{*}=\frac{r_{1} a_{21}-r_{2} a_{11}}{a_{11} a_{22}}>0, & \underline{y}^{*}=\frac{\left(a_{11} a_{22}-a_{12} a_{21}\right)\left(r_{1} a_{21}-r_{2} a_{11}\right)}{a_{11}^{2} a_{22}^{2}}>0 .
\end{array}
$$

Hence, by Theorem 2.5, we know that system (4.7) is persistent.

Choose constants $c_{1}$ and $d_{1}$ satisfying

$$
\frac{a_{22}}{a_{12}}>\frac{d_{1}}{c_{1}}>\frac{a_{21}}{a_{11}}
$$

Let $\alpha(\cdot)=c_{1}, \beta(\cdot)=d_{1}$ all be constant, then we have

$$
\left\{\begin{array}{l}
a_{11} d_{1}-a_{21} c_{1}>0 \\
a_{22} c_{1}-a_{12} d_{1}>0
\end{array}\right.
$$

Therefore assumption $(\mathrm{H} 4)$ of Theorem 3.1 holds. This completes the proof of Corollary 4.3.

Finally, we consider a Michaelis-Menten type functional response predator-prey system

$$
\left\{\begin{array}{l}
\dot{S}(t)=S(t)\left(1-\frac{S(t)}{K}-\frac{m x(t)}{a+S(t)}\right), \\
\dot{x}(t)=x(t)\left(\frac{m S(t)}{a+S(t)}-d-x(t)\right),
\end{array}\right.
$$

where $a, d, K$ and $m$ are all positive constants. We suppose that $K<a$ and $d<m$.

Letting $d \tau=a d(a+S) d t, \bar{x}=x(a-K) / K, \bar{S}=S(a-K) / a d K$, replace $d \tau$, $\bar{S}$ and $\bar{x}$ by $d t, S$ and $x$, respectively. Then we have

$$
\left\{\begin{array}{l}
\dot{S}(t)=S(t)\left(r_{1}-S(t)-a_{1} S^{2}(t)-m_{1} x(t)\right), \\
\dot{x}(t)=x(t)\left(-1+a_{2} S(t)-x(t) S(t)-m_{2} x(t)\right),
\end{array}\right.
$$


where $r_{1}=1 / d, a_{1}=a d K /(a-K)^{2}, a_{2}=K(m-d) /(a-K), m_{1}=m(a-K) / a d K$ and $m_{2}=(a-K) / d K$.

Letting $\bar{S}^{*}=2 r_{1} /\left(1+\sqrt{4 a_{1} r_{1}+1}\right), \bar{x}^{*}=\left(a_{2} \bar{S}^{*}-1\right) /\left(m_{2}+\bar{S}^{*}\right), r_{2}=r_{1}-m_{1} \bar{x}^{*}$, $\underline{S}^{*}=2 r_{2} /\left(1+\sqrt{4 a_{1} r_{2}+1}\right)$ and $\underline{x}^{*}=\left(a_{2} \underline{S}^{*}-1\right) /\left(m_{2}+\underline{S}^{*}\right)$, by Theorems 2.5 and 3.1, we obtain the following theorem.

THEOREM 4.4. If there is only one positive steady state in system (4.9), $\underline{S}^{*}>0$, $\bar{x}^{*}>0, \underline{x}^{*}>0$ and $a_{2} m_{1}<m_{2}$, then the positive steady state is globally attracting.

If $r_{1}=15, a_{1}=1 / 4, a_{2}=1 / 5, m_{1}=2$ and $m_{2}=4$, by simple computations we know that the equations

$$
\left\{\begin{array}{r}
15-S-S^{2} / 4-2 x=0 \\
-1+S / 5-S x-4 x=0
\end{array}\right.
$$

have only one positive solution. Moreover, we obtain

$$
\bar{S}^{*}=6, \quad \bar{x}^{*}=\frac{1}{50}, \quad \underline{S}^{*}=\frac{748}{25+5 \sqrt{399}}>\frac{29}{5}, \quad \underline{x}^{*}>\frac{2}{125} .
$$

The conditions of Theorem 4.4 then hold.

\section{Acknowledgement}

The authors would like to thank the referee for his/her valuable suggestions and comments. This project was supported by the NSFC of China (under Grant No. 10271044)

\section{References}

[1] S. Ahmad, "On almost periodic solutions of the competing species problems", Proc. Amer. Math. Soc. 102 (1988) 855-865.

[2] S. Ahmad, "On the nonautonomous Volterra-Lotka competition equations", Proc. Amer. Math. Soc. 177 (1993) 199-204.

[3] C. Alvarz and A. C. Lazer, "An application of topological degree to the periodic competing species problem", J. Austral. Math. Soc. Ser. B 28 (1986) 202-219.

[4] J. M. Cushing, "Two species competition in a periodic environment", J. Math. Biol. 10 (1980) $385-400$.

[5] J. M. Cushing, "Periodic Lotka-Volterra competition equations", J. Math. Biol. 24 (1986) 381-403.

[6] K. Gopalsamy, "Global asymptotic stability in a periodic Lotka-Volterra system", J. Austral. Math. Soc. Ser. B 27 (1985) 66-72. 
[7] K. Gopalsamy, "Global asymptotic stability in an almost periodic Lotka-Volterra system", $J$. Austral. Math. Soc. Ser. B 27 (1986) 346-360.

[8] C. Lansun, Mathematical models and methods in ecology, (in Chinese) (Science Press, Beijing, 1988).

[9] Z. Ma, Mathematic modeling and research of population ecology (An Fei Science Press, 1996).

[10] P. de Mottoni and A. Schiaffino, "Competition system with periodic coefficient: A geometric approach", J. Math. Biol. 11 (1981) 319-335.

[11] Z. D. Teng and L. S. Chen, "The positive periodic solutions of periodic Kolmogorov type systems with delays", Acta Math. Appl. Sinica 22 (1999) 446-456.

[12] J. H. Wu, X. Q. Zhao and X. Z. He, "Global asymptotic behavior in almost periodic Kolmogorov equations and chemostat models", Nonlin. World 3 (1996) 589-611.

[13] P. H. Yang and R. Xu, "Global attractivity of the periodic Lotka-Volterra system", J. Math. Anal. Appl. 233 (1999) 221-232.

[14] T. Yoshizawa, Stability theory and the existence of periodic solutions and almost periodic solutions (Springer, 1975). 